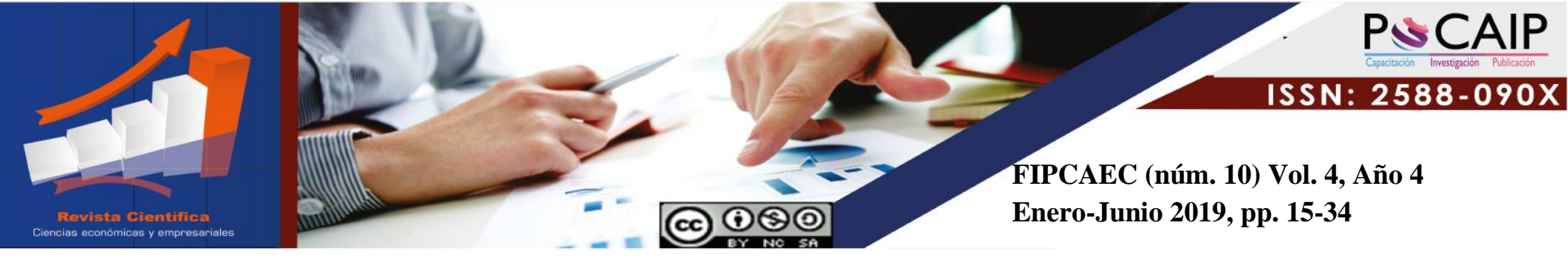

DOI: $10.23857 /$ fipcaec.v4i10.36

\title{
Una contemplación acerca de la Administración de empresas
}

\author{
A contemplation about Business Administration
}

\section{Uma contemplação sobre Administração de Empresas}

Michael Josué Suárez-Espinar ${ }^{1}$

josue201114v@gmail.com

https://orcid.org/0000-0003-1423-5081

Correspondencia: josue201114v@gmail.com

Recepción: 18/ 11/2018 * Aceptación: 12/12/2018 * Publicación: 15/01/2019

1 Investigador Independiente, Manta, Ecuador. 



\title{
Resumen
}

La inseguridad provocada por el proceso de la desintegración de los mercados y el aumento de la competencia implican grandes retos para las economías, tanto nacionales como regionales resultando de valiosa importancia el papel que juega la actividad empresarial y, en consecuencia, los empresarios y sus empresas. Teniendo en cuenta lo anterior, en el presente trabajo se describen algunos aspectos de relvada importancia relacionados con la administración de empresas, tales como: definición de empresa, clasificación, características, áreas funcionales, también estudia la administración empresas puntualizando las funciones de la administración y como punto desencadenante la planificación empresarial. Se concluye, la administración instituye una disciplina que se robustece cada vez más por ser indispensable en las organizaciones por su contribución al mejoramiento de las mismas a través de la implementación de las etapas del proceso administrativo logrando una mayor competitiva en su entorno.

Palabras clave: Empresa; administración; planificación empresarial; efectividad, eficiencia.

\begin{abstract}
The insecurity caused by the process of market disintegration and increased competition entail major challenges for both national and regional economies, the role played by business activity and, consequently, businessmen and their companies. Taking into account the above, in this paper some aspects of important importance related to business administration are described, such as: business definition, classification, characteristics, functional areas, business administration also studies highlighting the functions of administration and as a trigger point business planning. It concludes, the administration institutes a discipline that is increasingly strengthened by being indispensable in organizations for their contribution to improving them through the implementation of the stages of the administrative process, achieving greater competitiveness in their environment.
\end{abstract}

Keywords: Company; administration; business planning; effectiveness, efficiency 


\section{Resumo}

A insegurança causada pelo processo de desintegração do mercado e o aumento da concorrência envolvem grandes desafios para as economias nacional e regional, o papel desempenhado pela atividade empresarial e, consequentemente, pelos empresários e suas empresas. Considerando o exposto, neste artigo são descritos alguns aspectos de importância importante relacionados à administração de empresas, tais como: definição, classificação, características, áreas funcionais, administração de empresas, além de estudos que destacam as funções de administração e administração. Como um planejamento de negócios de ponto de gatilho. Conclui que a administração institui uma disciplina cada vez mais fortalecida, sendo indispensável nas organizações por sua contribuição para melhorá-las através da implementação das etapas do processo administrativo, alcançando um ambiente competitivo mais competitivo.

Palavras-chave: Empresa; administração; planejamento de negócios; eficácia, eficiência

\section{Introducción}

Se conceptualiza la empresa como "el agente que organiza con eficiencia los factores económicos para producir bienes y servicios para el mercado, con el ánimo de alcanzar ciertos objetivos, entre los que destaca el beneficio empresarial. (Pozo Rodríguez, J M, Martins Kidito X Gama, M, \& Rodríguez Cotilla, Z. 2014), López Carvajal, G.; F. Becerra y R. Pino 2007)

Es notable acotar que la empresa funciona bajo el principio del autofinanciamiento, lo que indica que no solo cubrirá sus gastos con sus ingresos, sino que además generará un margen de utilidades. Se adscribe directamente a un órgano superior de dirección empresarial, o al órgano estatal o de gobierno que se determine. (Pozo Rodríguez, J M, Martins Kidito X Gama, M, \& Rodríguez Cotilla, Z. 2014)

Según se ha citado el dinamismo empresarial está asentado en desafiar oposiciones y de aprovechar las oportunidades en los mercados concibiendo riqueza y empleo en el territorio. De tal forma, las claves tanto del avance como del atraso relativo de un territorio pueden encontrarse en la fortaleza o fragilidad de su tejido empresarial. (Fernández Serrano. J. 2012) 
Es de importancia referirnos que en la década de los setenta se planteó el avance del desarrollo económico a largo plazo por lo que germinaron decisiones que originaron la aparición de empresas como mecanismo básico para dinamizar las economías, flexibilizar el tejido productivo y acelerar el cambio estructural. Hoy en día principales organismos e instituciones internacionales, entienden la puesta en marcha de políticas de promoción de pequeñas y medianas empresas (PYMEs) que se pueden aproximar por su tamaño y funcionamiento a la figura del empresario individual como medio de garantizar la adaptación y competitividad de los sistemas económicos de los países al nuevo contexto de globalización internacional; la promoción del espíritu empresarial en las políticas de desarrollo local se ha establecido como un elemento primordial para el avance de cualquier economía.( Fernández Serrano. J. 2012)

Cabe señalar que entre los investigadores en estas dos últimas décadas el análisis del Entrepreneurship y su relación con el desarrollo y el crecimiento económico adquirió una gran importancia. (Audretsch y Keilbach, 2005); (Plummer y Acs, 2005); (Audretsch., 2006).

Se destaca que cada una de las empresas ejecuta actividades disímiles, poseen plantillas totalmente distintas y se estructuran según sus necesidades por lo que el tejido empresarial que sacude la economía está formado por multitud de empresas, compañías y organizaciones de diferentes características que poco tienen que ver unas con otras.

\section{Clasificación de las empresas}

\section{Empresas según su tamaño}

Según el número de trabajadores y el tipo de estructura que posean podemos diferenciar estos tipos de empresas:

- Microempresas: son empresas que tienen hasta un máximo de 10 trabajadores y suelen pertenecer a un único socio que también trabaja para la empresa. Muchas de empresas tienen gran potencial y pueden desarrollarse en empresas más grandes si se invierte en ellas 
- Pequeñas empresas: las pequeñas empresas poseen un número de trabajadores que va desde los 11 hasta los 49. Muchas de estas empresas son negocios familiares y ya poseen una estructura organizacional que deriva en una división del trabajo. Suelen ser empresas rentables e independientes, aunque no poseen grandes recursos financieros y de capital.

- Medianas empresas: las pequeñas y medianas empresas son gran parte de la economía y el tejido empresarial. Estas últimas poseen plantillas de entre 50 y 250 trabajadores con una estructura y departamentos organizados que permiten delimitar el trabajo y las responsabilidades.

- Grandes empresas: este tipo de empresas poseen más de 250 trabajadores y en la mayoría de ocasiones apuestan en la internacionalización con el objetivo de llevar sus productos por todo el mundo y conseguir mayores beneficios

Según su forma jurídica está determinada por el número de socios, capital, y tipo de responsabilidad de cada una de las personas dueñas de la empresa.

- Empresas individuales: si solo pertenece a una persona. Esta puede responder frente a terceros con todos sus bienes, es decir, con responsabilidad ilimitada, o sólo hasta el monto del aporte para su constitución, en el caso de las empresas individuales de responsabilidad limitada o EIRL. Es la forma más sencilla de establecer un negocio y suelen ser empresas pequeñas o de carácter familiar.

- Empresas societarias o sociedades: constituidas por varias personas. Dentro de esta clasificación están: la sociedad anónima, la sociedad colectiva, la sociedad comanditaria y la sociedad de responsabilidad limitada

- Las cooperativas u otras organizaciones de economía social. (Tipos de empresas. 2017).

\section{Según la actividad económica que desarrolla}

- Industriales: La actividad primordial de este tipo de empresas es la producción de bienes mediante la transformación de la materia o extracción de materias primas. Las industrias, a su vez, se clasifican en: 
- Extractivas: Cuando se dedican a la explotación de recursos naturales, ya sea renovables o no renovables. Ejemplos de este tipo de empresas son las pesqueras, madereras, mineras, petroleras, etc.

- Manufactureras: Son empresas que transforman la materia prima en productos terminados, y pueden ser:

- De consumo final: Producen bienes que satisfacen de manera directa las necesidades del consumidor. Por ejemplo: prendas de vestir, alimentos, aparatos eléctricos, etc.

- De producción: Estas satisfacen a las de consumo final. Ejemplo: maquinaria ligera, productos químicos, etc.

Comerciales: Son intermediarias entre productor y consumidor; su función primordial es la compra/venta de productos terminados. Pueden clasificarse en:

- Mayoristas: Venden a gran escala o a grandes rasgos.

- Minoristas (detallistas): Venden al menudeo.

- Comisionistas: Venden de lo que no es suyo, dan a consignación.

Servicios: Son aquellas que brindan servicio a la comunidad que a su vez se clasifican en:

- Transporte

- Turismo

- Instituciones financieras

- Servicios públicos (energía, agua, comunicaciones)

- Servicios privados (asesoría, ventas, publicidad, contable, administrativo)

- Educación

- Finanza

- Salubridad, entre otras. ( Definición de empresa y su clasificación . 2015)

\section{La Clasificación de las Empresas también se pueden dar según el Ámbito de Operación:}

- Empresas locales

- Regionales

- Nacionales

- Multinacionales 


\section{Según la Composición del Capital:}

- Empresa privada: si el capital está en manos de particulares.

- Empresa pública: si el capital y el control está en manos del Estado.

- Empresa mixta: si la propiedad es compartida.

- Empresa de autogestión: si el capital está en manos de los trabajadores. (Definición y Clasificación de las Empresas.2010)

Según la cuota de mercado que poseen las empresas: (Definición y Clasificación de las Empresas.2010)

- Empresa aspirante: aquélla cuya estrategia va dirigida a ampliar su cuota frente al líder y demás empresas competidoras, y dependiendo de los objetivos que se plantee, actuará de una forma u otra en su planificación estratégica.

- Empresa especialista: aquélla que responde a necesidades muy concretas, dentro de un segmento de mercado, fácilmente defendible frente a los competidores y en el que pueda actuar casi en condiciones de monopolio. Este segmento debe tener un tamaño lo suficientemente grande como para que sea rentable, pero no tanto como para atraer a las empresas líderes

- Empresa líder: aquélla que marca la pauta en cuanto a precio, innovaciones, publicidad, etc., siendo normalmente imitada por el resto de los actuantes en el mercado.

- Empresa seguidora: aquélla que no dispone de una cuota suficientemente grande como para inquietar a la empresa líder.

Literaturas estudiadas hacen referencia que la característica de una empresa está dada por los elementos que la componen, los cuales las relacionadas a continuación:

- Factores activos: empleados, propietarios, sindicatos, bancos, etc.

- Factores pasivos: materias primas, transporte, tecnología, conocimiento, contratos financieros, etc.

- Organización: coordinación y orden entre todos los factores y las áreas. 


\section{Factores activos}

Personas físicas y/o jurídicas (entre otras entidades mercantiles, cooperativa, fundaciones, etc.) que constituyen una empresa realizando, entre otras, aportación de capital (sea puramente monetario, sea de tipo intelectual, patentes, etc.). Estas "personas" se convierten en accionistas de la empresa.

Participan, en sentido amplio, en el desarrollo de la empresa:

- Administradores.

- Clientes.

- Colaboradores y compañeros.

- Fuente financiera.

- Suministradores y proveedores.

- Trabajadores.

\section{Factores pasivos}

Todos los que son usados por los elementos activos y ayudan a conseguir los objetivos de la empresa. Como la tecnología, las materias primas utilizadas, los contratos financieros de los que dispone, etc.

\section{Áreas funcionales de una empresa}

Es expresado que las áreas funcionales son las divisiones que se realizan en una empresa con la finalidad de lograr su mejor funcionamiento, por lo que el éxito de una empresa consiste en la correcta coordinación de las diversas áreas funcionales.

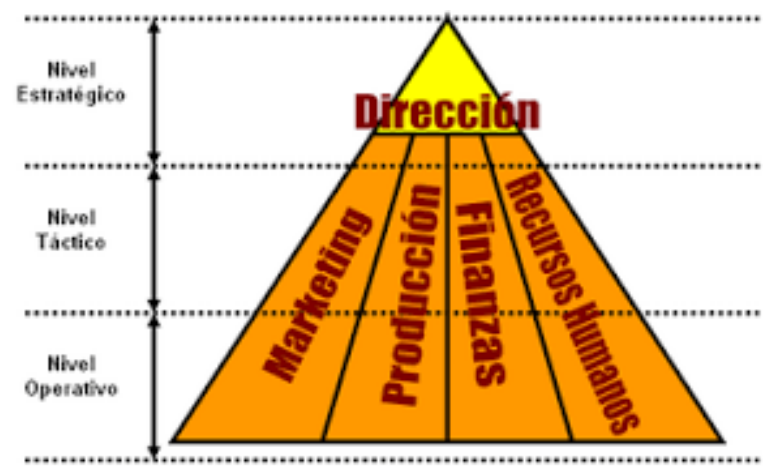

Fuente: Administración: Wikipedia la enciclopedia libre 


\section{La administración en las empresas}

La contribución acopiada de diversos precursores, filósofos, físicos, economistas, estadistas y empresarios que, con el paso del tiempo, fueron desarrollando y divulgando cada uno, obras y teorías en su campo de actividades da surgimiento a la administración.( Introducción a la administración. 2018)

Reyes Ponce A. (2018) Define en su investigación que la palabra administración es la « función de lograr que las cosas se realicen por medio de otros » u obtener resultados a través de otros » Alistamos diferentes definiciones expresadas por importantes estudiosos de la temática: (Celaya Osorio Y. 2018)

Mario Sverdlik: "es la coordinación de todos los recursos a través del proceso de planeación, dirección y control, a fin de lograr objetivos establecidos.

Robert F. Buchele: «El proceso de trabajar con y a través de otras personas a fin de lograr objetivos, de una organización formal.»

Harold Koontz y Cyril O`Donnell: "Es la dirección de un organismo social y su efectividad en alcanzar sus objetivos, fundada en la habilidad de conducir a sus integrantes.

Isaac Guzmán Valdivia: «Es la dirección eficaz de las actividades y la colaboración de otras personas con el fin de obtener determinados resultados.»

George R. Terry: "Consiste en lograr un objetivo predeterminado, mediante el esfuerzo ajeno.»

José A. Fernández Arena: «Es una ciencia social que persigue la satisfacción de objetivos institucionales por medio de una estructura y a través del esfuerzo humano coordinado.»

Es primordial destacar que la administración en los momentos actuales es reverenciada por sus contribuciones al proceso de desarrollo de la humanidad como un fenómeno universal; a nivel global se le reconoce por instaurarse como una actividad distinguida que se fundamenta en el esfuerzo cooperativo que el hombre desarrolla en las organizaciones públicas y privadas, en una 
sociedad caracterizada por una creciente complejidad en sus relaciones, así como una mayor dinámica en los procesos de cambio, transformación y adecuaciones de las cuestiones administrativas que tienen que ver con el progresivo flujo de bienes, servicios, personas y dinero que se realizan al interior de cada país y a través de las fronteras nacionales.(Méndez Sánchez $V$ M, Méndez Ortiz L , Cruz Landa A J (2018):

A lo largo de los planteamientos hechos, es esencial señalar, que la administración en su situación actual, ha transcurrido por un proceso evolutivo en el que cada etapa de su historia, desde el surgimiento de la administración científica, se caracteriza por el desarrollo de los avances teóricos, herramientas y metodologías que las necesidades y problemáticas específicas de cada época han requerido. (Méndez Sánchez V M, Méndez Ortiz L, Cruz Landa A J 2018):

\section{Funciones de la administración}

\section{Planificación}

Es el proceso que comienza con la visión de la organización; la misión de la organización; fijar objetivos, las estrategias y políticas organizacionales, usando como herramienta el Mapa estratégico; todo esto teniendo en cuenta las fortalezas/debilidades de la organización y las oportunidades/amenazas del contexto (Análisis FODA). La planificación abarca el largo plazo (de 5 años a 10 o más años), el mediano plazo (entre 1 años y 5 años) y el corto plazo donde se desarrolla el presupuesto anual más detalladamente.

\section{Organización}

Responde a las preguntas de, ¿Quién? va a realizar la tarea, implica diseñar el organigrama de la organización definiendo responsabilidades y obligaciones; ¿cómo? se va a realizar la tarea; ¿cuándo? se va a realizar; mediante el diseño de Proceso de negocio, Cursogramas que establecen la forma en que se deben realizar las tareas y en que secuencia temporal; en definitiva organizar es coordinar y sincronizar. 


\section{Dirección}

Es la persuasión, que se ejerce por medio del Liderazgo sobre los individuos para la consecución de los objetivos fijados; basado esto en la toma de decisiones usando modelos lógicos y también intuitivos de Toma de decisiones.

\section{Control}

Es la medición del desempeño de lo ejecutado, comparándolo con los objetivos y metas fijados; se detectan los desvíos y se toman las medidas necesarias para corregirlos. El control se realiza a nivel estratégico, nivel táctico y a nivel operativo; la organización entera es evaluada, mediante un sistema de Control de gestión; por otro lado también se contratan auditorías externas, donde se analizan y controlan las diferentes áreas funcionales de la organización. (Celaya Osorio Y. 2018)

\section{Retroalimentación}

Se evidencia de acuerdo a las funciones de la administración; ayuda a tomar decisiones con juicios y sapiencia, muestra un panorama de lo moral y no moral de lo correcto y no correcto; la toma de decisiones en una empresa es de mucha responsabilidad e importancia para los administradores.

Resumiendo: La administración es el proceso de trabajar con las personas con los recursos para alcanzar los objetivos de la empresa de forma eficaz y eficiente.

Manifestaremos de forma breve algunos aspectos a tener en cuenta en la planificación empresarial.

Se especifica que la planificación empresarial es un proceso técnico, económico y organizativo que beneficia a las empresas a encausar sus acciones en el cumplimiento de sus objetivos a corto y mediano plazos, además de estas ideas, la planificación es un proceso que expresa el interés de realizar un uso adecuado de los recursos disponibles y la voluntad de priorizar el aporte de las empresas estatales a la sociedad, por encima de cualquier interés colectivo o individual. 
La planificación por ende la elaboración del plan es un asunto continuo de interacción entre los diferentes actores económicos para lograr determinados objetivos en el tiempo, a partir de la constante retroalimentación de las proyecciones o los cambios que se puedan producir en el entorno internacional, nacional y/o empresarial.

En general la planificación, y el sistema de planificación empresarial, en particular, tienen que ser vistos con un enfoque temporal llamado "horizonte de planificación", cuya necesidad objetiva se deriva del hecho de que las tareas económicas anuales (plan empresarial) están sujetas a objetivos a mediano plazo, es decir, la planificación debe garantizar la correspondencia entre los intereses del desarrollo futuro con las especificidades y características del presente.

El proceso de planificación o de elaboración del plan de la empresa debe ser: continuo, integrador, participativo, flexible, reflexivo, dinámico y de mejora continúa:

- Continuo: el proceso de elaboración del plan es ininterrumpido, que se organiza por etapas, y que desde los primeros meses del año anterior al que se planifica, se comienzan a realizar los estudios de demanda, capacidad, entre otros, para conformar el plan del siguiente año.

- Integrador: cuando el área económica, en el proceso de elaboración del plan, integra la información que recibe de las demás áreas funcionales de la organización para conformarlo.

- Participativo: activa participación de los directivos de las áreas funcionales: mercadotecnia, recursos humanos, operaciones, finanzas y economía, del personal técnico de estas áreas y de todos los trabajadores que laboran en la organización.

- Flexible: pueden cambiar las condiciones en las que fue elaborado el plan, en tanto, es una guía de acción; luego se debe tener la capacidad para incorporar las afectaciones que pueden tener cambios en los precios, costos de los insumos o la posibilidad de responder a variaciones no previstas de la demanda.

- Reflexivo: documento que permita el espacio para el análisis y el debate científico con la amplia participación de todos. Cualquier idea que implique racionalidad, debe ser escuchada, valorada e incorporada. 
habilidades interpersonales; facilidades en campos como las matemáticas, contabilidad y finanzas; y análisis estratégico.

Opina el autor que no existe una empresa sin su correspondiente anchura administrativa.

\section{La administración de empresa en América Latina}

En la última década en América Latina, los Centro Desarrollo Empresarial han tenido un lanzamiento muy importante y en la actualidad una quincena de países de la región ha dado vida a políticas nacionales para la promoción de estos centros, logrando movilizar enormes cantidades de recursos de los sectores público, académico y privado. Sin embargo, en la trama de este diverso escenario, los Centros de Desarrollo Empresarial mantienen ciertos elementos comunes que les permiten destacar positivamente entre las políticas de apoyo de las MIPYMES latinoamericanas. (Dini M 2019)

- En primer lugar, representan un intento para institucionalizar la acción de apoyo a las MIPYMES, proyectándola en el mediano y largo plazo.

- En segundo lugar, se esfuerzan para introducir un modelo de gestión orientado a los resultados y una disciplina de evaluación permanente de los impactos generados en las empresas atendidas.

- En tercer lugar, se desarrollan a partir de un esfuerzo conjunto de distintos sectores de la sociedad: el sector público, el académico y el empresarial, que colaboran aportando recursos y conocimientos para el desarrollo de las MIPYMES.

- En cuarto lugar, proponen una modalidad de atención integral y un acompañamiento a las empresas beneficiarias que permite asistirlas a lo largo de sus procesos de transformación.

- Por último, operan de una manera descentralizada, adaptándose a las realidades de los territorios en los que se localizan. .(Dini M 2019)

Según Castillo, Gligo, \& Rovira, (2017).Cito (...) algunos países de la región están en las primeras etapas del proceso de implementación de los centros; otros están revisando el esquema adoptado hasta el momento e, incluso los que ya cuentan con una estructura más desarrollada, están preguntándose cómo consolidarla y cómo ajustarla a los desafíos que plantea la revolución industrial en curso . 
Investigaciones desarrolladas por la Comisión Económica para América Latina y el Caribe (CEPAL), arrojan que en América Latina las MIPYMES representan aproximadamente el 99\% de los establecimientos; son responsables de alrededor del $61 \%$ del empleo formal y del $25 \%$ del valor añadido; y su aporte a las exportaciones se coloca, generalmente, entre el 2 y el $10 \%$. Existe, obviamente, variaciones importantes por país y por sector, pero en términos generales la contribución de las MIPYMES a la economía regional en estas cuatro variables se ha mantenido en los rangos mencionados, en el periodo analizado.(Dini M 2019)

El horizonte empresarial que impera en la región, está acreditado por una heterogeneidad extremadamente manifiesta entre agentes productivos $\mathrm{y}$, en particular, por una fuerte polarización entre las empresas micro que son responsables de aproximadamente dos tercios de los empleos formales y las grandes que generan, por si solas, alrededor del 75\% de la producción (Correa, Leiva, \& Stumpo, 2018)

Ecuador

Tello Caicedo, G E y colaboradores (2018) en su investigación concerniente a la responsabilidad social empresarial corporativa y su incidencia en el ámbito laboral en Ecuador hacen referencia que es una institución privada sin fines de lucro aprobada en dic.2005 por el Ministerio de Cultura de la República del Ecuador, impulsa la práctica, la aplicación técnica y metodológica de la responsabilidad social con base en la implantación del modelo de RSE, mediante el uso de herramientas universales, así como también que los directivos de las empresas en el desarrollo de sus estrategias permitan el desarrollo de personas, el bienestar de la sociedad, la sostenibilidad del medio ambiente .

En último lugar; en la actualidad la administración instituye una disciplina que se robustece cada vez más por ser indispensable en las organizaciones por su contribución al mejoramiento de las mismas a través de la implementación de las etapas del proceso administrativo logrando una mayor competitiva en su entorno, para esto es necesario tener en cuenta la planeación desde la visión de la mejora continua, Aldana-Zavala (2017), Aldana \& Piña (2017), Isea-Argüelles \& Aldana-Zavala (2017), esto con la finalidad de promover el conocimiento de las fortalezas y 
debilidades de la empresa en pro de promover estrategias favorecedoras de su crecimiento integral.

\section{Referencias}

1. Aldana, J., \& Piña, J. (2017). Calidad del servicio prestado al cliente por los instructores de gimnasios. Revista Arbitrada Interdisciplinaria Koinonía, 2(3), 172-197. Recuperado de http://fundacionkoinonia.com.ve/ojs/index.php/revistakoinonia/article/view/59/46

2. Aldana-Zavala, J.J. (2017). Modelo de gestión gerencial basado en el modelo racional para la toma de decisiones. Revista Científica FIPCAEC (Fomento De La investigación Y publicación En Ciencias Administrativas, Económicas Y Contables). ISSN: 2588-090X. Polo De Capacitación, Investigación Y Publicación (POCAIP), 2(4), 31-48. https://doi.org/10.23857/fipcaec.v2i4.30

3. Audretsch, D.B., Bönte, W. y Keilbach, M. (2005): Regional entrepreneurship capital and its impact on knowledge Diffusion and Economic Performance, Working Paper del Group Entrepreneurship, Growth and Public Policy, Alemania: Max- Planck Institute of Economics.

4. Celaya Osorio Y. (2018). La importancia de la Administración de Empresas. Recuperado de: https://www.emprendices.co/la-importancia-de-la-administracion-de-empresas/

5. Castillo, M., Gligo, N., \& Rovira, S. (2017). La política industrial 4.0 en América Latina. In Políticas industriales y tecnológicas en América Latina. Santiago de Chile.

6. Correa, F., Leiva, V., \& Stumpo, G. (2018). Mipyme y heterogeneidad estructural en América Latina. In MIPYME en América Latina: un frágil desempeño y nuevos desafíos para las políticas de fomento. Santiago de Chile: CEPAL.

7. DEFINICION DE EMPRESA Y SU CLASIFICACIÓN. 2015 Recuperado de : https://www.academia.edu/7535000/DEFINICION_DE_EMPRESA_Y_SU_CLASIFICA $\mathrm{CI} \% \mathrm{C} 3 \% 93 \mathrm{~N}$

8. Definición y Clasificación de las Empresas.2010. Recuperado de : https://encolombia.com/economia/empresas/definicionyclasificaciondelaempresa 
16. Plummer, L.A. y Acs, Z.J. (2005): - Penetrating the Knowledge Filter in regional economiesll, Annals of Regional Science, 39: 439- 456.

17. Reyes Ponce A. Administración moderna. 2018. Recuperado de : https://educativoinsurgentes.files.wordpress.com/2018/01/administracion-moderna-reyesponce.pdf

18. Tipos de empresas. 2017. Recuperado de : https://www.emprendepyme.net/tipos-deempresas

19. Tello Caicedo, G E, Agila Maldonado, M V, \& Legarda Arreaga, C. (2018). La responsabilidad social empresarial corporativa y su incidencia en el ámbito laboral en Ecuador. Revista Universidad y Sociedad, 10(5), 60-69. Epub 02 de diciembre de 2018. Recuperado de http://scielo.sld.cu/scielo.php?script=sci_arttext\&pid=S2218$36202018000500060 \& \operatorname{lng}=$ es\&tlng=es.

\section{References}

1. Aldana, J., \& Piña, J. (2017). Quality of service provided to the client by gym instructors. Interdisciplinary Arbitrated Review Koinonía, 2 (3), 172-197. Recovered from http://fundacionkoinonia.com.ve/ojs/index.php/revistakoinonia/article/view/59/46

2. Aldana-Zavala, J.J. (2017). Management management model based on the rational model for decision making. FIPCAEC Scientific Magazine (Promotion of research and publication in Administrative, Economic and Accounting Sciences). ISSN: 2588-090X. Training, Research and Publication Center (POCAIP), 2 (4), 31-48. https://doi.org/10.23857/fipcaec.v2i4.30

3. Audretsch, DB, Bönte, W. and Keilbach, M. (2005): Regional entrepreneurship capital and its impact on knowledge Diffusion and Economic Performance, Working Paper of the Group Entrepreneurship, Growth and Public Policy, Germany: Max- Planck Institute of Economics

4. Celaya Osorio Y. (2018). The importance of Business Administration. Recovered from: https://www.emprendices.co/la-importancia-de-la-administracion-de-empresas/ 
13. López Carvajal, G.; F. Becerra and R. Pino (2007). «Business planning», Faculty of Economic and Business Sciences, University of Cienfuegos,

14. Méndez Sánchez V M, Méndez Ortiz L, Cruz Landa A J (2018): “The importance of administration in organizations as a way to achieve competitiveness", Caribbean Journal of Social Sciences (January 2018). Recovered from: :http://www.eumed.net/rev/caribe/2018/01/administracion-organizaciones.html

15. Pozo Rodríguez, J M, Martins Kidito X Gama, M, \& Rodríguez Cotilla, Z. (2014). The logic of business planning and work productivity. Economy and Development, 152 (2), 122-137. Retrieved from http://scielo.sld.cu/scielo.php?script=sci_arttext\&pid=S0252$85842014000200008 \& \operatorname{lng}=$ en $\&$ tlng=en.

16. Plummer, L.A. and Acs, Z.J. (2005): - Penetrating the Knowledge Filter in regional economies\|, Annals of Regional Science, 39: 439-456.

17. Reyes Ponce A. Modern administration. 2018. Recovered from: https://educativoinsurgentes.files.wordpress.com/2018/01/administracion-moderna-reyesponce.pdf

18. Types of companies. 2017. Recovered from: https://www.emprendepyme.net/tipos-deempresas

19. Tello Caicedo, G E, Agila Maldonado, M V, \& Legarda Arreaga, C. (2018). Corporate corporate social responsibility and its impact on the workplace in Ecuador. University and Society Magazine, 10 (5), 60-69. Epub December 2, 2018. Retrieved from http://scielo.sld.cu/scielo.php?script=sci_arttext\&pid=S2218$\underline{36202018000500060 \& \operatorname{lng}=\mathrm{en} \& \operatorname{tlng}=\mathrm{en}}$.

(C)2019 por el autor. Este artículo es de acceso abierto y distribuido según los términos y condiciones de la licencia Creative Commons Atribución-NoComercial-Compartirlgual 4.0 Internacional (CC BY-NC-SA 4.0)

(https://creativecommons.org/licenses/by-nc-sa/4.0/). 